\title{
Tác giả Việt nêu quan điểm về việc rút bài báo khoa học trên Nature
}

\author{
Hồ Mạnh Toàn \\ Khoa học \& Phát triên \\ 09/06/2020 15:03
}

KH\&PT (https://khoahocphattrien.vn/khoa-hoc/tac-gia-viet-neu-quan-diem-ve-viec-rutbai-bao-khoa-hoc-tren-nature/20200609100930455p1c160.htm) - Tác giả Vương Quân Hoàng (Trung tâm Nghiên cứu Xã hội Liên ngành ISR, trường Đại học Phenikaa) vừa có bài viêt quan điểm trên tạp chí Nature về việc làm thế nào để việc rút bài báo khoa học trở nên minh bạch hơn, mang lại lợi ích cho bản thân nhà khoa học bị rút bài cũng như cộng đồng khoa học.

\section{Comment}

\section{Retraction-Hydroxychloroquine or chloroquine with or without a macrolide for treatment of COVID-19: a multinational registry analysis}

\footnotetext{
After publication of our Lancet Article, ${ }^{1}$ several concerns were raised with respect to the veracity of the data and analyses conducted by Surgisphere Corporation and its founder and our co-authoc, Sapan Desai, in our publication. We launched an independent third. party peer review of Surgisphere with the consent of Sapan Desai to evaluate the origination of the database elements, to confirm the completeness of the database. and to replicate the analyses presented in the paper.

Our independent peer reviewers informed us that Surgisphere would not transfer the full dataset, client contracts, and the full ISO audit report to their servers for analysis as such transfer would violate client agreements and confidentiality requirements. As such, our reviewers were not able to conduct an independent and private peer review and therefore notified us of their withdrawal from the peer-review process.

We always aspire to perform our research in accordance with the highest ethical and professional guidelines. We can never forget the responsibility we have as researchers to scrupuloushy ensure that we rely on data sources that adhere to our high standards. Based on this development, we can no longer vouch for the veracity of the primary data sources. Due to this unfortunate development the authors request that the paper be retracted.
}

We all entered this collaboration to contribute Musuletoment in good faith and at a time of great need during the COVID-19 pandemic. We deeply apologise to you, the editors, and the joumal readership for any embarrassment or inconvenience that this may have caused.

MisM mports perenenal fees from Abbott. Medtronic Januen Rowant.

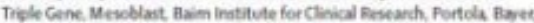
Nupulsecl. Finctleart and leviticus frir has been paid for time spent 35 a

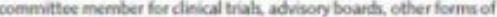
consulting and lectures or presentations these parmentswere made diectly

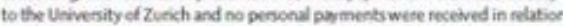
to these trialsor other at tivities since 2018 Before 2018 FR repoits orants and

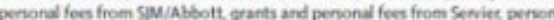
foes from Zoll personal fees from Astra Zeneca peroonel fees from Sroch.

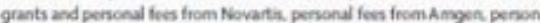

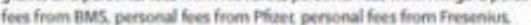
penond fes from Vifoc penond fes frem Pxhe orats and penond feses

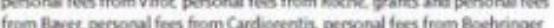

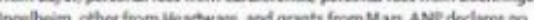
competing interests.

"Mandeep R Mehra, Frank Ruschitzka. Amit N Patel mmehraebwh. harvard edu

Brigham andW Womenis Hospital Heat andVasodar Center and Hanard Medical

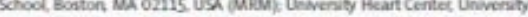

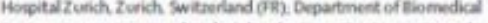
Engineering Undennity of Utah, Salt Lake Ciex UT, USA (ANF) and HCA Reseanch institute Nashivile TN USA (ANE)

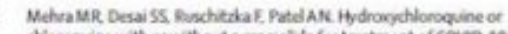

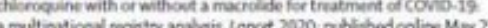

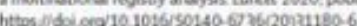


Nghiên cứu về dùng Hydroxychloroquine hoặc Chloroquine trong điều trị COVID-19 trên tạp chí y khoa hàng The Lancet vừa bi rút lại vi không chứng minh được nguồn gốc và độ tin cậy của dữ liệu. Ảnh: The Lancet Twitter

Mới đây, hai nghiên cứu trên hai tạp chí y khoa hàng đâuu là The Lancet và New

England Journal of Medicine (NEJM) bị rút lại do các nhà khoa học không chứng minh được nguồn gốc và độ tin cậy của dữ liệu. Trong đó, bài báo trên The Lancet sử dụng dữ liệu được cung cấp bởi công ty Surgisphere để thực hiện nghiên cứu lâm sàng về dùng Hydroxychloroquine hoặc Chloroquine trong điều trị COVID-19.

Bài báo trên NEJM cũng sử dụng dữ liệu được cung cấp bởi cùng công ty để phân tích về bệnh tim mạch và phương pháp điều trị trong thời kì COVID-19. Ở cả hai bài báo, các tác giả đã không thể tiếp cận và xác nhận được dữ liệu do Surgisphere cung cấp. Giữa thời kì COVID-19, các nhà khoa học đều cố gắng đóng góp các tri thức mới cho nhân loại nhanh nhất có thể, vì thế, họ cũng khó tránh khỏi các sai lầm rất "người", và đôi lúc, nó dẫn tới hệ quả bị rút bài.

Liên quan đến vấn đề rút bài báo khoa học, tác giả Vương Quân Hoàng (Trung tâm Nghiên cứu Xã hội Liên ngành ISR, trường Đại học Phenikaa) vừa có bài viết quan điểm trên tạp chí Nature.

Dựa trên kết quả phân tích 2.000 thông báo rút bài được đăng tải trên tạp chí Learned Publishing [2018 Impact Factor = 2.2; 2018 CiteScore $=1.89$ ], tác giả Vương Quân Hoàng cho biết, có rất ít thông tin mà người đọc nhận được từ các thông báo này. Vì vậy, tác giả đề nghị các tạp chí và cộng đồng khoa học cần đảm bảo đủ 4 thông tin sau đây trong mỗi thông báo rút bài: ai bắt đầu việc rút bài; nguyên nhân; việc rút bài có sự đồng thuận giữa biên tập và tác giả không; và việc xuất bài có đóng góp từ quá trình phản biện sau xuất bản không.

Việc nêu rõ và đầy đủ các thông tin này có thể mang lại nhiêu lợi ích cho nhà khoa học bị rút bài cũng như cộng đồng khoa học. Trước hết, nó cung cấp đủ thông tin giúp nhà khoa học được cảm thông vì sự sơ suất hơn là sự kỳ thị vì việc rút bài. Điều này cũng giúp các nhà khoa học dũng cảm hơn trong việc nhận lỗi. Ngoài ra, sự minh bạch còn giúp cộng đồng hiểu thêm về khoa học: mỗi kết quả đều dựa trên nhiều lần thử nghiệm và sai lầm.

Để đạt được sự minh bạch này, bên cạnh việc áp dụng thông báo rút bài với 4 phần, tạp chí khoa học cũng có thể để mở phần giới hạn của nghiên cứu. Điều này giúp thay đổi quan niệm rằng chỉ có các nghiên cứu "sạch" và "xác quyết" mới là một nghiên cứu quan trọng.

Việc rút bài làm lộ ra những khiếm khuyết và cả sai phạm trong nghiên cứu. Mặc dù điều này thường để lại những ấn tượng không tốt đẹp nhưng thực tế, nó cho thấy cơ chế kiểm định chất lượng, phát hiện lỗi đang làm việc hiệu quả. Vì thế, theo tác giả 
Vương Quân Hoàng, chúng ta nên thay đổi quan niệm về việc rút bài, nó đơn giản chỉ là một cách thức thực tế để con người sửa sai và làm khoa học ngày một tốt đẹp hơn.

\section{* Tham khảo:}

Vuong QH. (2020). Reform retractions to make them more transparent. Nature 582(7811):149. doi: 10.1038/d41586-020-01694-x. URL:

https://www.nature.com/articles/d41586-020-01694-x.

Vuong QH. (2020). The limitations of retraction notices and the heroic acts of authors who correct the scholarly record: an analysis of retractions of papers published from 1975-2019. Learned Publishing 33(2):119-130; doi:10.1002/LEAP.128. URL: https://onlinelibrary.wiley.com/doi/full/10.1002/leap.1282. 